\title{
The lateral stiffness and strength required to ensure membrane action at the ultimate load of a reinforced concrete slab-and-beam floor*
}

\author{
by R. Park, M.E., Ph.D., A.M.I.Struct.E.
}

\section{Contribution by Professor R. G. Robertson, M.A., M.I.C.E., M.I.Struct.E. Professor of Civil Engineering, University of Cape Town}

Dr Park seems to imply that interior slabs are the only ones that could carry enhanced loading due to membrane action. This was not found to be so in the 50 full-scale tests made at Alliance House, Cape Town, in 1957. The ultimate load for outer corner slabs was three-quarters of that for the inner slabs (except when the inner slabs had initial cracks, found to be present in all floors, diagonally across two bays at each corner of the building), the inner slabs carried about four times, and the corner (and the cracked slabs) three times, the load calculated by the yield-line theory.

Dr Park's statement that more steel is required when membrane compression is used to support a load is also open to question; the slab compression does not increase the tension in the beam steel but reduces the compression at the top of the beam; this compression is transferred to the slab as in $T$ beam action, without altering the beam steel required, and hence compressive membrane action in the slab enables the slab reinforcement to be reduced by the total amount of beam steel present, which usually gives a significant saving.

This last statement may alternatively be worded that the beam reinforcement can be obtained free: this economy can be achieved by reducing the slab steel required by yield-line theory in the slab, and placing this steel in the beams whose depth may be chosen so that this amount of steel will be fully utilized as beam steel. Thus compressive membrane action is far from being of " academic" value only, as Dr Park stated.

Some 30 years ago I realized that square slabs gave the greatest economy for decking and designed such slabs for deck structures for viaducts and wharves in London. I had developed a logical analysis and formula for the behaviour of the cracked slab, after yielding, which fits subsequent published test results extremely well and is given below.

My equation for unreinforced square slabs gives the ultimate load per unit area as

$p=\frac{3 K_{1} K_{3} u}{l^{2}}\left(1-K_{2}\right) Z\left(Z+\frac{\xi l_{2}}{1-K_{2}}\right)\left(\frac{2 d-Z}{Z+\xi \frac{l}{2}}\right)^{2}$.

where $l=$ span, $d=$ depth, $u=$ cube strength, $Z=$

*Pages 29 to 38 of Magazine No. 50.

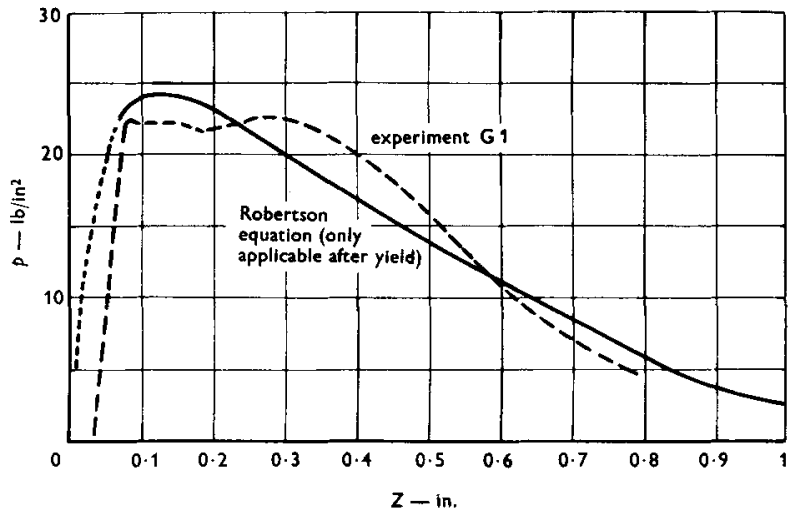

Figure I: Membrane action in slabs. The broken curve is reproduced from Figure 9 of the paper by Park.

central deflexion and

$$
\xi=\frac{K_{1} K_{3} u}{2}\left(\frac{1}{K}+\frac{l}{2 E_{c} d}\right) \ldots \ldots \ldots
$$

where $K_{1}, K_{2}, K_{3}$, are the ultimate load stress coefficients as given by the author, $K$ is the abutment stiffness (force per unit length of abutment for unit lateral displacement) and $E_{r}$ is the elastic modulus of concrete.

For a single point-load, the ultimate value is $W=$ $(\pi / 12) p L^{2}$. Generally it could be assumed in equation 2 that $K=4 A_{s} E_{s} / l^{2}$ where $A_{s}=$ total section area of beam steel. The value of $K$ should be larger for interior slabs with uncracked surrounds.

Taking the author's test slab $G_{1}$ :

$A_{s}=\frac{1}{8}$ in $^{2}, l=12$ in., $d=0.69$ in., $K=100,000 \mathrm{lb} / \mathrm{in}^{2}$, $u=2,490 \mathrm{lb} / \mathrm{in}^{2}$.

Assume $E_{c} \dagger=3 \times 10^{6} \mathrm{lb} / \mathrm{in}^{2}, K_{1} K_{3}=0.67, K_{2}=0.48$. From equation 2 ,

$$
\xi=830\left(\frac{1}{100,000}+\frac{6}{3 \times 10^{6} \times 0 \cdot 69}\right)=0.0107
$$

From equation 1,

$$
p=18 Z(Z+0 \cdot 12)\left(\frac{1 \cdot 38-Z}{Z+0 \cdot 064}\right)^{2}
$$

†See CP 115 (1959), Clause 304C. 


\begin{tabular}{c|c|c|c|c|c|c|c|c|c|c}
\hline$Z$ & $0 \cdot 01$ & 0.02 & 0.05 & $0 \cdot 1$ & $0 \cdot 2$ & $0 \cdot 3$ & $0 \cdot 4$ & $0 \cdot 6$ & $0 \cdot 8$ & $1 \cdot 0$ \\
\hline$p$ & 8 & 12 & 20 & 24 & 23 & 20 & 17 & 11 & 6 & $2 \cdot 6$ \\
\hline
\end{tabular}

It is seen that the above equation closely represents the test results given in Figure 9 of the author's text, reproduced in Figure $I$.

In the Cape Town experiments, tests were made on 20 models and 50 full-scale slabs and the expansion of the abutments was measured; in all cases, it would have been interesting if Dr Park had done so in his tests.

\section{Reply by the author}

I did not wish to imply that the exterior panels of slab-and-beam floors could not carry enhanced ultimate loads due to membrane action. I commented in the paper that in an exterior panel the membrane forces developed in the direction normal to the outside edge would be small because the edge beam alone would have to provide the lateral restraint. With panels of small reinforcement content, however, even small membrane forces will considerably enhance the ultimate load. The difficulty in calculating the ultimate load of exterior panels is in estimating the reduction in the membrane action occurring as a result of the lateral bowing of the edge beams. The test results quoted by Professor Robertson are of interest in indicating the extent of this effect in his floors.

The compressive membrane forces acting in a slab must be balanced by tension in the supporting beams, otherwise membrane action could not be enforced. The line of action of this tensile force will lie in the flange of each $\mathrm{T}$ beam and the beams need to be designed to carry flexure and tension. Professor Robertson is incorrect in stating that the beams do not require extra steel to carry this tension. In the regions of the beam where the bending moment is hogging, the tension reinforcement in the top of the beam for flexure will approximately coincide with the line of action of the membrane force to be balanced and if, for instance, the tensile force to be carried due to membrane action is equal to the force in the tension steel, the beam will have no flexural strength at all in this region. Therefore a large amount of extra steel for tie reinforcement (equal to the membrane force to be balanced) is required in addition to the reinforcement for flexure in the regions of hogging bending moment in the beam. In the region of sagging bending moment in the beam, it is true that, provided the tensile force in the reinforcement in the beam for flexure exceeds the membrane force to be carried, no extra steel is required in that portion of the beam since the membrane force is carried by a reduction in the compression at the top of the beam. However, extra steel will have to be placed where the sagging moment is small, because the compressive force in the beam will be inadequate. Also, it must be remembered that the membrane force to be balanced is much greater than the yield force of the steel saved from the panel. When all these factors are taken into account it is apparent that the quantity of extra steel required in the beams for tie reinforcement may exceed that saved in the panel. Certainly if tie reinforcement is placed continuously in the beams to balance all the membrane forces (as in the slabs with ring steel tested by the author) the amount of tie reinforcement required will greatly exceed that saved in the panels.

Professor Robertson's theory for the load-deflexion curves is of interest although no indication of the manner of the derivation of his equations is given. My theory is also based on a load-deflexion relationship which gives a curve rising to maximum load and then falling away with increasing deflexion. A difficulty with this theory (and with that of Professor Robertson) is that full plasticity is assumed at the yield lines at all deflexions and no indication is given of the deflexion at which it becomes applicable. Therefore, there is no guarantee that the slab when tested will follow the early part of this theoretical load-deflexion curve. Hence, the maximum load given by the theoretical curve may not be the ultimate load because the deformation of the slab may be insufficient to develop the yield lines at that deflexion. In order to determine the ultimate load from the theoretical load-deflexion relationship, I have had to resort to test results. A large number of tests has shown that panels in stiff surrounding frames reach ultimate load at a central deflexion which is generally not greater than 0.5 of the slab depth. This is a criterion which needs improvement but, since a large deflexion should give a conservative value for the ultimate load, equations 1 and 2 of the paper are the load at a central deflexion of $0.5 d$. If, in the theory of reference 3 and in the paper, the central deflexion is left as a variable instead of being put equal to $0 \cdot 5 d$, expressions may be derived which give the load carried at any central deflexion assuming full plasticity at the yield lines. For an unreinforced square interior panel with continuous tie steel carrying all the ring tension, the load-deflexion relationship so obtained may be shown to be:

$$
\begin{aligned}
& w=\frac{k_{1} k_{3} u d^{2}}{L^{2}}\left\{12\left(1-k_{2}\right)+3 \frac{\Delta}{d}\left(2 k_{2}-3\right)+\left(\frac{\Delta}{d}\right)^{2}\left(2-k_{2}\right)\right. \\
& +\left(\varepsilon+\frac{2 t}{L}\right) \frac{L^{2}}{d^{2}}\left(3 \frac{d}{\Delta}\left(2 k_{2}-1\right)+1 \cdot 5\left(1-k_{2}\right)\right) \\
& \left.-0 \cdot 75 k_{2}\left(\varepsilon+\frac{2 t}{L}\right)^{2} \frac{L^{4}}{d^{2} \Delta^{2}}\right\} \\
& \text { where } \varepsilon=\frac{4-\frac{\Delta}{d}-\frac{2 t L}{d \Delta}}{\frac{8 E_{c}}{k_{1} k_{3} u}+\frac{L^{2}}{d \Delta}}
\end{aligned}
$$




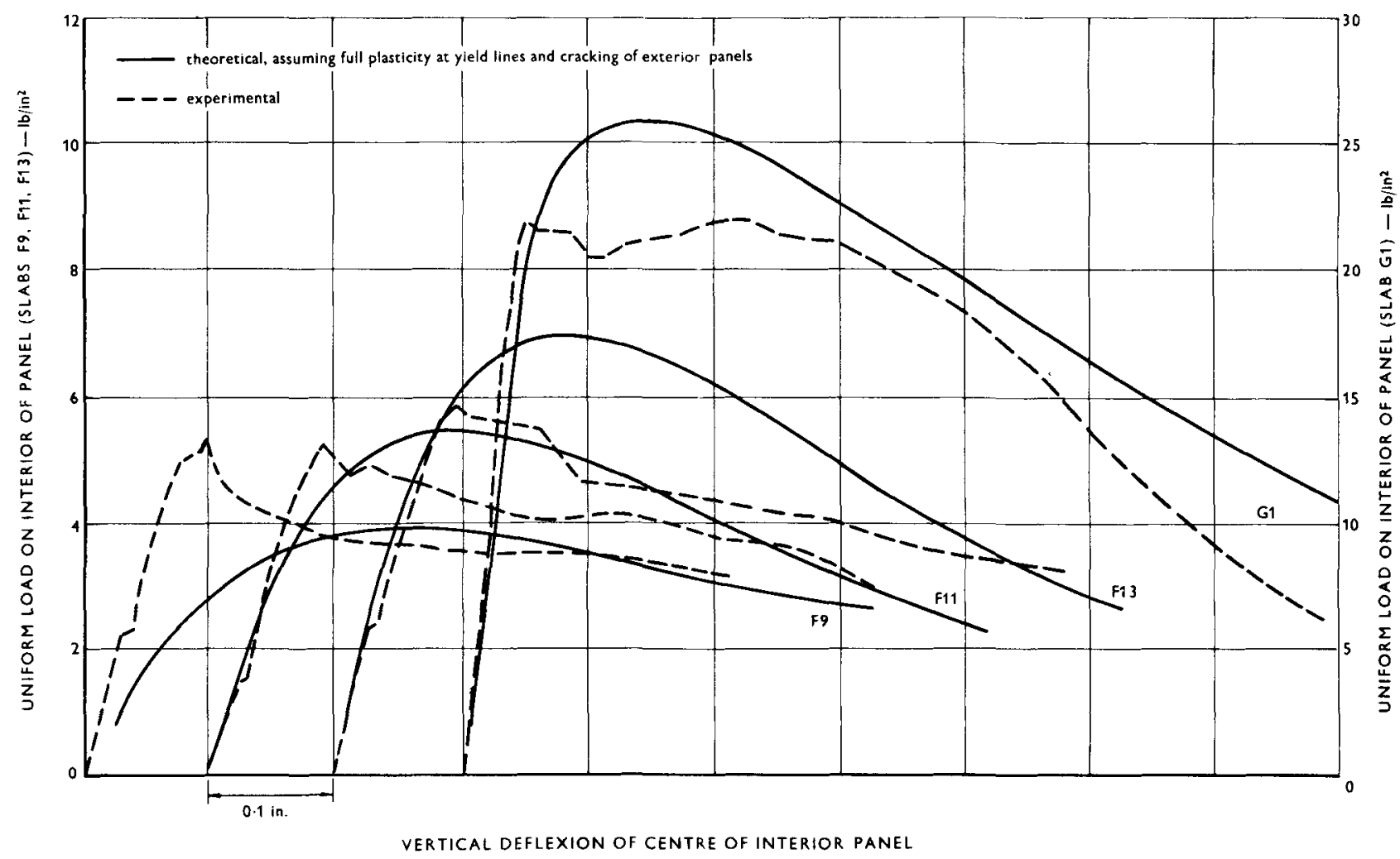

Figure II: Comparison of theoretical and experimental load-deflexion curves.

and $t=\frac{\left(4-\frac{\Delta}{d}\right)\left(\frac{8 E_{c}}{k_{1} k_{3} u}+\frac{L^{2}}{d \Delta}\right)-\left(\frac{4 L^{2}}{d \Delta}-\frac{L^{2}}{d^{2}}\right)}{\left(\frac{32 E_{s} A_{t}}{k_{1} k_{3} u d L_{t} L}+\frac{2 L}{\Delta d}\right)\left(\frac{8 E_{c}}{k_{1} k_{3} u}+\frac{L^{2}}{d \Delta}\right)-\frac{2 L^{3}}{d^{2} \Delta^{2}}}$

where $\delta=$ central deflexion and the other notation is as defined in the paper.
The load-deflexion curves given by these equations are plotted in Figure II against the experimental results of the interior panels of slabs with square exterior panels and continuous ring steel around the panels. Good agreement is shown to be obtained only at large deflexions, the discrepancy at small deflexions being due mainly to the lack of full development of plasticity and the stage of cracking of the exterior panels. 